Invited Reviews for the 2008 Hirosi Kuriyama Award

\title{
Pathophysiology underlying irritable bowel syndrome -From the viewpoint of dysfunction of autonomic nervous system activity-
}

\author{
Noriaki MANABE ${ }^{1}$, Toshiaki TANAKA ${ }^{2}$, Jiro HATA ${ }^{1}$, \\ Hiroaki KuSUNOKI $^{3}$ and Ken HARUMA ${ }^{2}$ \\ ${ }^{1}$ Division of Endoscopy and Ultrasonography, Department of Clinical Pathology and \\ Laboratory Medicine; \\ ${ }^{2}$ Division of Gastroenterology, Department of Internal Medicine; \\ ${ }^{3}$ Department of Health Care Medicine; Kawasaki Medical School, Japan
}

Received December 1, 2008; Accepted December 10, 2008

\begin{abstract}
Although irritable bowel syndrome (IBS) is the most common gastrointestinal disease and is found in up to $50 \%$ of patients referred to gastroenterologists, its pathogenesis remains unexplained. Lately most attention has been focused on visceral hypersensitivity related to dysfunction of the autonomic nervous system. Although there have been many reports regarding the autonomic nervous system in IBS patients, the results have not always been consistent. In many studies, increased sympathetic nervous system activity and decreased parasympathetic nervous system activity have been the most frequently noted differences when IBS patients are compared with healthy controls. There are also some differences among IBS symptom subgroups and some differences between men and women. Data from previous studies have been inconsistent, with complicating factors resulting in difficulties in classification and the possibility of multiple physiological pathways for a similar symptom picture. Further studies based on the Rome III criteria are required to evaluate whether or not autonomic functions significantly differ from each other. However, although there are many methods of measurement for evaluation of the autonomic nervous system, there are few of these methods that are not only accurate but also cost-effective, non-invasive, and easy to perform. Recently, we developed a new method with the abovementioned advantages for measuring fingertip blood flow in evaluating the autonomic nervous system by continuous-wave Doppler sonography. Using this method, we found that abnormal fingertip blood flow responses suggested the presence of excess sympathetic activity.
\end{abstract}

Key words: irritable bowel syndrome, sympathetic nervous system, parasympathetic nervous system, continuous-wave Doppler sonography

Correspondence to: Noriaki Manabe, MD, Divison of Endoscopy and Ultrasonography, Department of Clinical Pathology and Laboratory Medicine, Kawasaki Medical School, 577, Matsushima, Kurashiki 7010192, Japan Phone: +81-86-462-1111 Fax: +81-86-462-1199 e-mail: n_manabe@hkg.odn.ne.jp 


\section{Introduction}

Irritable bowel syndrome (IBS) is a functional disorder that affects $10 \%$ to $20 \%$ of the population worldwide (Pan et al., 2000; Ringel et al., 2001; Gwee et al., 2004). IBS also accounts for $12 \%$ of visits to primary care physicians and $28 \%$ of referrals to gastroenterologists (Mitchell et al., 1987). In a survey of health service utilization, the odds of a patient with IBS incurring charges were 1.6-fold greater than for a similar asymptomatic individual, which translated into overall median yearly charges of \$742 in 1992 dollars for each patient with IBS compared to \$429 for controls (Talley et al., 1995).

IBS produces health consequences in addition to its economic costs. IBS produces disability rates equal to or greater than those for severe organic gastrointestinal (GI) disease and is the second leading cause of absenteeism after the common cold, with patients with IBS missing three times as many work days yearly as persons without IBS (Schuster et al., 1991; Drossman et al., 1993; Walker et al., 1995).

IBS is characterized by altered bowel habits and abdominal discomfort in the absence of organic disease. No clear diagnostic markers exist for IBS and thus diagnoses are based on the clinical presentation. The Rome III criteria for diagnosis of IBS include at least three months of continuous or recurrent abdominal pain or discomfort that has two or more following features: improvement with defecation, association with a change in the frequency, and a change in stool form (George et al., 2006).

Inappropriate treatment for patients leads to poor patient satisfaction. Understanding the pathophysiological mechanisms underlying these conditions has, therefore, become an important matter in treating them. However, the pathogenesis of IBS is poorly understood. At least two etiological mechanisms, altered bowel motility and increased visceral sensitivity, have been proposed to account for the GI symptoms observed in patients with IBS (Mayer et al., 1994). It has been suggested that the autonomic nervous system can modulate visceral sensitivity and that the central nervous system can influence the motility and secretory activity of the GI tract by way of autonomic pathways via the enteric nervous system (Iovino et al., 1995; Vandler et al., 1998). It has also been suggested that subtle abnormalities in the autonomic nervous system are an underlying factor in the symptoms reported by patients with functional GI disorders (Bockus et al., 1928; Bharucha et al., 1993).

For these reasons, the autonomic nervous system is considered to play an important role in the pathophysiology of IBS. In this report, we will first review autonomic dysfunction in patients with IBS, and then we will introduce a new method for evaluating autonomic nerve function that we recently developed.

\section{Modulation of the autonomic nervous system and patients' response to stress}

Physiological stimuli in the gut elicit appropriate reflex responses to perform the digestive function (Malagelada et al., 1989). Normally, the digestive process evolves unperceived, but under some circumstances, patients may experience abdominal symptoms. IBS is characterized by abdominal pain or discomfort combined with alterations in bowel function. As early as 1928, 
Table 1. Previous studies investigating autonomic nervous system function in IBS patients

\begin{tabular}{|c|c|c|c|}
\hline Study & Number & Method & Findings (vs. controls) \\
\hline $\begin{array}{l}\text { Karling et al. } \\
\text { (1998) }\end{array}$ & $\begin{array}{l}18 \text { IBS } \\
36 \text { controls }\end{array}$ & $\begin{array}{l}\text { HRV lying in the supine position } \\
\text { and } 70^{\circ} \text { head up position }\end{array}$ & $\mathrm{SNS} \uparrow, \mathrm{PNS} \rightarrow$ \\
\hline $\begin{array}{l}\text { Adeyemi et al } \\
\text { (1999) }\end{array}$ & $\begin{array}{l}35 \text { IBS } \\
18 \text { controls }\end{array}$ & $\begin{array}{l}\text { HRV at rest, orthostatic stress, } \\
\text { in the deep breathing mode }\end{array}$ & $\begin{array}{l}\text { SNS at rest } \uparrow, \text { PNS suppression during orthostatic stress } \downarrow \\
\text { Increased PNS in the deep breathing mode } \downarrow\end{array}$ \\
\hline $\begin{array}{l}\text { van Orshoven } \\
(2006)\end{array}$ & $\begin{array}{l}\text { et al. } \\
18 \text { IBS } \\
19 \text { controls }\end{array}$ & $\begin{array}{l}\text { Blood pressure, heart rate, } \\
\text { HRV, and MSNA before, during } \\
\text { and after consumption of a } \\
\text { standardized meal }\end{array}$ & $\mathrm{PNS} \downarrow, \mathrm{MSNA} \rightarrow, \mathrm{SNS} \uparrow$ \\
\hline $\begin{array}{l}\text { Tanaka et al. } \\
\text { (2007) }\end{array}$ & $\begin{array}{l}59 \text { IBS } \\
40 \text { controls }\end{array}$ & $\begin{array}{l}\text { FTBF before, during, and after } \\
\text { cold stress }\end{array}$ & $\mathrm{SNS} \uparrow$ \\
\hline $\begin{array}{l}\text { Spaziani et al. } \\
\text { (2008) }\end{array}$ & $\begin{array}{l}39 \text { IBS } \\
98 \text { controls }\end{array}$ & $\begin{array}{l}\text { heart rate and blood pressure } \\
\text { before, during and after the } \\
\text { rectal distension using BRS }\end{array}$ & BRS $\downarrow$, blood pressure response $\uparrow$ \\
\hline
\end{tabular}

HRV: heart rate variability, SNS: sympathetic nervous system, PNS: parasympathetic nervous system. MSNA: muscle sympathetic nerve activity. FTBF: fingertip blood flow, BRS: baroreceptor sensitivity.

it was hypothesized that dysfunction of the autonomic nervous system contributed to the symptoms seen in IBS (Bockus et al., 1928). The sympathetic and parasympathetic branches of the autonomic nervous system mediate central nervous system communication with the gut, largely through modulation of the third autonomic nervous system branch, the enteric nervous system. Through its three divisions, the autonomic nervous system modulates and coordinates GI motility, secretion, and immune function (Elenkov et al., 2000; Hansen, 2003). Modulation of the autonomic nervous system, including increased sympathetic nervous system and decreased parasympathetic nervous system activity, is an important part of the organism's response to stress. The resulting changes in GI function may provide the mechanism linking life stress and affective states to IBS symptoms.

\section{Autonomic nervous system function in IBS patients}

Some studies have investigated autonomic nervous system function in patients with IBS. Of these, the main ones are shown in Table 1. Results have not always been consistent, possibly due to differences in sample characteristics, assessment parameters, and conditions under which measurements were taken. As might be expected, increased sympathetic nervous system activity and decreased parasympathetic nervous system activity were the most frequently noted differences when IBS patients were compared with healthy controls (Table 1). Recently we reported on autonomic nervous system dysfunction in patients with IBS using fingertip blood flow as measured by laser Doppler flowmetry (Tanaka et al., 2007). We found that IBS patients had increased sympathetic nervous system activity, which is consistent with previous studies. Diarrhea-predominant patients with IBS show an increased level of anxiety and high excretion 
Table 2. Subtyping IBS by predominant stool pattern

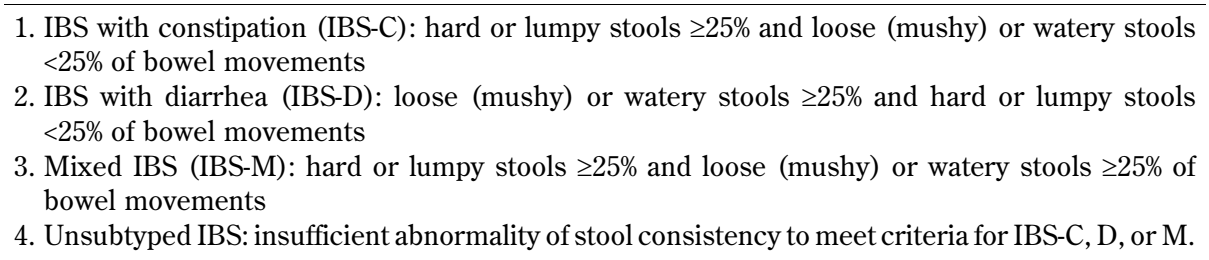

of free epinephrine in urine (Esler et al., 1973). Heitkamper et al. reported high levels of catecholamines and cortisol in urine in women with IBS (Heitkemper et al., 1996). These data are consistent with the above reports, including ours, that patients with IBS have increased sympathetic nervous system activity. Patients with IBS are known to have a higher incidence of mood disturbances, anxiety, depression, somatization disorders, and psychologic distress (Drossman et al., 1977). Therefore, one explanation for the increased sympathetic activity in patients with IBS might be increased psychologic arousal and distress. Sympathetic activation has been shown to increase perception of intestinal distention (Iovino et al., 1995), which might explain the frequent abdominal pain and distention reported by patients with IBS.

Although whether these autonomic nerve dysfunctions are a result or cause of the pathogenesis of IBS is an important issue, we can not answer exactly for it. If autonomic nerve dysfunction is a result of IBS, one would expect to see more autonomic nerve dysfunction associated with a longer duration of IBS. However, no significant correlation has been reported between the duration of IBS and severity of autonomic nerve functions.

\section{Differences in autonomic function among IBS symptom subgroups}

According to recent Rome III criteria, IBS is divided into four subtypes based on predominant stool pattern (Table 2). However, there have been a few reports regarding the difference in autonomic function among the IBS subtypes according to this criterion. Since almost all previous reports were based on the Rome II criteria, in this report, we review the difference in autonomic function among the IBS subtypes according to the Rome II. The Rome II criteria classified IBS into three subgroups, a diarrhea-predominant type, a constipation-predominant type and a painpredominant type (Drossman et al., 2002). Some studies reported differences in the autonomic activity of these IBS subgroups. Aggarwal et al. found the vagal dysfunction of IBS patients to be particularly associated with constipation-predominant symptoms, whereas they found adrenergic sympathetic dysfunction to be especially associated with diarrhea-predominant symptoms (Aggarwal et al., 1994). Elsenbruch et al. (2001) reported that IBS diarrhea-predominant patients differed from constipation-predominant IBS patients in their postprandial cardiac autonomic and cortisol responses. Namely, diarrhea-predominant IBS patients were shown to have autonomic and cortisol hyper-responsiveness unlike that of constipation-predominant IBS patients and controls. Robert et al. (2006), on the other hand, observed elevated sympathetic dominance and vagal withdrawal during non-REM and REM sleep in diarrhea-predominant IBS patients, but not in those with alternating type IBS. However, constipation-predominant IBS could not be 
distinguished from diarrhea-predominant IBS or alternating type IBS with regard to autonomic activity. They concluded that there might be a continuum of autonomic dysfunction among these symptom subgroups. Tillish et al. (2005) reported the same results as Robert et al. (2006). Our data (Tanaka et al., 2007) were also consistent with these reports. Data from previous studies has been inconsistent regarding bowel habit effect, with the complicating factors being difficulties in classification and possible multiple physiological pathways for a similar symptom picture. Further studies based on the Rome III criteria should be carried out to evaluate whether or not autonomic function in IBS subgroups differs significantly.

\section{Sex specific alterations in autonomic function among IBS patients}

Generally women are diagnosed with IBS two to three times more often than men in western countries (Everhart et al., 1991). In addition, women make up $80 \%$ of the population with severe IBS (Longstreth et al., 1993). Recent evidence has confirmed that sex differences exist among IBS patients in terms of perception (Chang et al., 2001), central response to visceral stimuli (Naliboff et al., 2003), and colonic transit response to pharmacological treatment (Viramontes et al., 2003). Tillisch et al. (2005) reported that the autonomic differences seen in IBS appeared to be particularly due to changes in male subjects. They also found that male IBS patients had significantly higher sympathovagal balance than healthy male controls, whereas no differences were noted between IBS females and female controls. But some reports have been in disagreement with the above findings. However, differences in autonomic nervous system responses to specific stimuli may also play a role in producing sex-based variations in IBS symptom patterns and in differential responses to some pharmacological agents (Chang et al., 2002; Mayer et al., 2004).

\section{IBS patients often have cutaneous hyperalgia}

In humans, the sympathetic nervous system modulates perception and reflex responses. Increased sympathetic nervous system activity and decreased parasympathetic nervous system activity are the most frequently noted signs in IBS patients, which leads to the possibility of visceral hypersensitivity in IBS patients. We often observed cutaneous hyperalgia in IBS patients (Tanaka et al., 2007). The present study could not determine exactly whether or not this was a result of impaired extrinsic gut nerve activity, but this finding is supported by several previous studies. Mayer et al. reported that patients with IBS also exhibited a number of extraintestinal manifestations such as migraine headache, back pain, heartburn, dyspareunia and muscle pain consistent with the central hyperalgesic mechanism (Mayer et al., 1994).

\section{Association between cardiac autonomic nerve function and abdominal autonomic nerve function}

There have been many reports regarding autonomic nerve function in patients with IBS, but they used methods for measuring several cardiac autonomic nerve functions, such as beat-to-beat 
heart rate and blood pressure variation in response to deep breathing, postural changes, the Valsalva maneuver, and cold stress. Nowadays, spectral analysis of heart rate variability is available as a modern computerized techniques to evaluate cardiac autonomic nerve function. Although these measures of autonomic nervous system activity do not directly provide information on the role of the autonomic nervous system in gastrointestinal function, Buysschaert et al. (1985) reported a close association between cardiac and gastric autonomic function. Additionally, Emmanuel and Kamm found that cardiovagal tone, as measured by heart rate variability, correlates with rectal mucosal blood flow and colonic transit, further supporting the relationship between cardioautonomic measures and gastrointestinal function (Emmanuel et al., 2000).

\section{New method for measuring fingertip blood flow response to evaluate autonomic nerve function}

From the aforementioned point of view, the autonomic nervous system may be considered to play an important role in the pathophysiology of IBS. There have been many good methods for evaluating autonomic nerve function, but they have been required the active cooperation of patients, time or cost-consuming with consequent difficulties in tenderization. At present, laser Doppler flowmetry (LDF), which assesses microcirculatory blood flow in the skin to a depth of about $1 \mathrm{~mm}$, is the most commonly used method for assessing peripheral autonomic dysfunction (Abe et al., 1996; Shiihara et al., 1999; Raamat et al., 2002). Carried out at the fingertips, this technique can be used under resting conditions and also during phasic sympathetic activation by means of deep inspiratory gasps and brief electrical stimuli as well as during tonic activation by sustained painful stimuli (cold presser test) or by mental arithmetic. With ongoing progress in ultrasonographic techniques, continuous-wave (cw) Doppler sonography is now increasingly being used to measure microcirculatory blood flow. This method is cost-effective, non-invasive, easy to perform and, most importantly, readily available in the vast majority of facilities. We developed a new method for measuring fingertip blood flow to evaluate autonomic nervous function by cw Doppler sonography.

The experimental protocol is described in detail below. The study was performed after overnight fasting. All subjects were instructed to refrain from smoking, eating and drinking after 9 PM the previous night. First, the subjects rested in the supine position for approximately 30 min to become acclimated. Room temperature was kept uniform at $24^{\circ} \mathrm{C}$. At the end of the acclimatization period, we confirmed that the skin temperature at the fingertips as measured by an infrared thermometer was above $30^{\circ} \mathrm{C}$ in all subjects. Fingertip blood flow in the second finger of the right hand was assessed by cw Doppler sonography. After baseline measurement for at least $5 \mathrm{~min}$, the subjects received sympathetic stimulation from cold stress applied without notification in the form of an ice bag $\left(0^{\circ} \mathrm{C}\right)$ upon the left forearm for $1 \mathrm{~min}$. The fingertip blood flow response was measured for $5 \mathrm{~min}$. A representative time-velocity waveform is shown schematically in Fig. 1. After at least $5 \mathrm{~min}$, fingertip blood flow velocity was stable and was taken as the value of $\mathrm{V}_{\text {pre. }}$. After cold stress, the velocity of fingertip blood flow decreased sharply within a few minutes $\left(T_{1}\right)$, and then remained near the minimal velocity obtained $\left(V_{\min }\right)$ for about a minute $\left(T_{2}-T_{3}\right)$. During the recovery period $\left(T_{3}\right)$, fingertip blood flow velocity gradually 


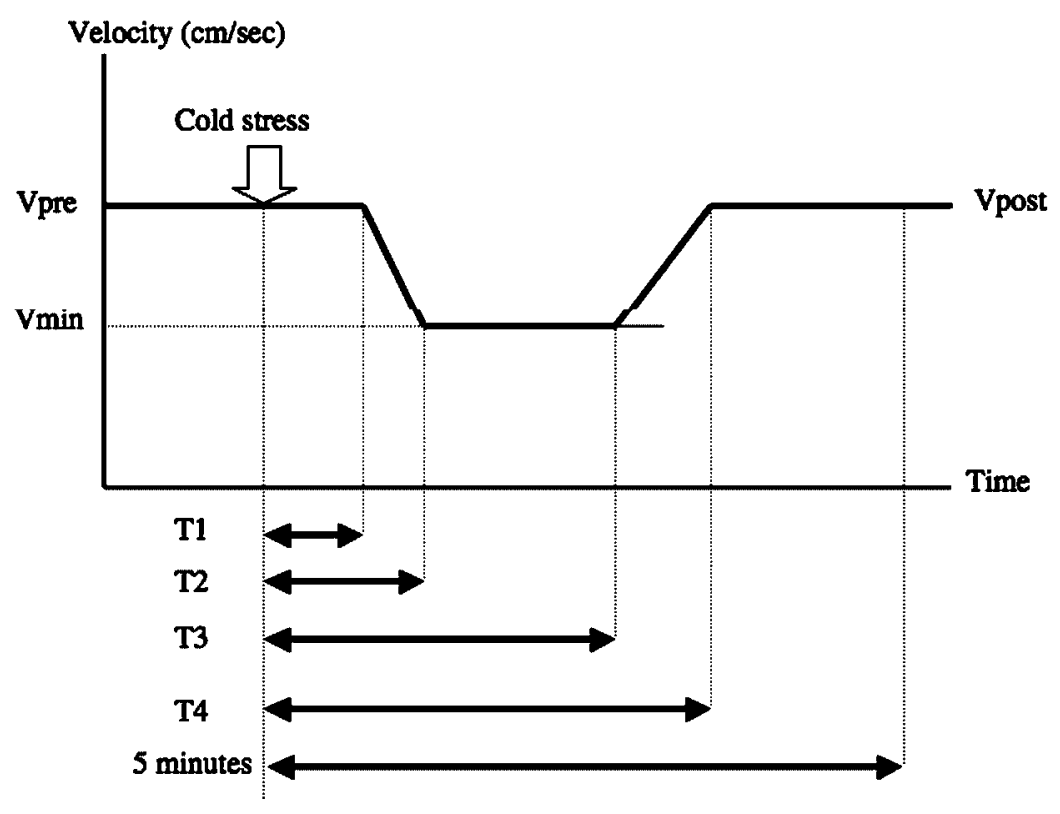

Fig. 1. A representative time-velocity waveform, shown schematically.

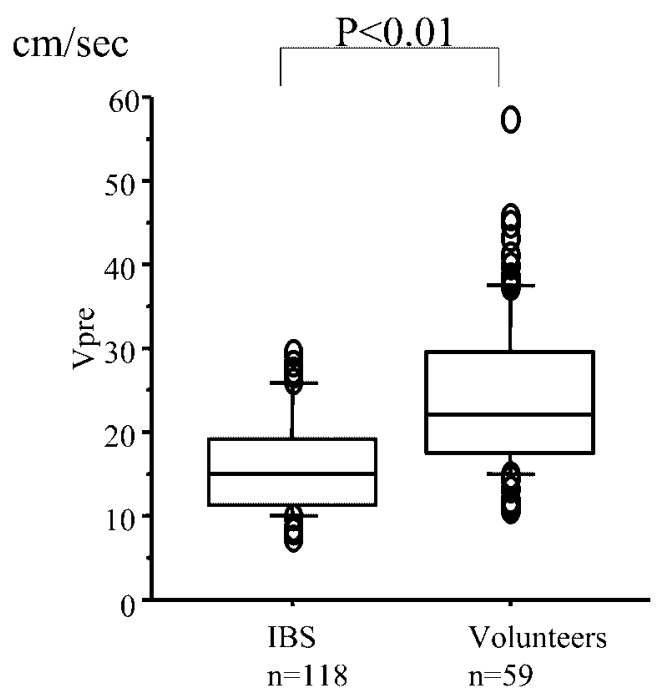

Fig. 2. Comparison of fingertip blood flow velocity at the baseline between the irritable bowel syndrome and the control subjects using continuous-wave Doppler ultrasonography. $\mathrm{V}_{\text {pre }}$ was significantly lower in the IBS patients than in the control subjects $(P<0.01)$.

increased, returning to the velocity at the baseline $\left(\mathrm{V}_{\text {post }}\right)$ at the time point designated $\mathrm{T}_{4}$. Using this method, we demonstrated that the fingertip blood flow of patients with IBS had significantly lower $\mathrm{V}_{\text {pre }}$ and $\mathrm{V}_{\min }$, a significantly longer $\mathrm{T}_{4}$, and a significantly lower fingertip blood flow response than that of the control subjects, reflecting increased sympathetic activity (Fig. 2). 


\section{Closing remarks}

There is sufficient evidence to conclude that IBS is an important medical disorder with significant impact on those afflicted with regard to symptom severity, disability, and impaired quality of life. Furthermore, the burden to society in terms of direct health care costs and the indirect effects including work absenteeism, exceeds that of most gastrointestinal disorders. To date, no single shared etiologic factor has been found in patients with IBS. In recent years, attention has been drawn to the relationship between abnormal brain-gut interaction and the pathogenesis of functional gastrointestinal disorders. Nowadays, it is generally recognized that abnormal brain-gut interactions play an important role in IBS. The autonomic nervous system in humans may mediate abnormal brain-gut interaction like the sympathetic nervous system modulates perception and reflex responses to gut distention. Although some studies seem to indicate such a relationship, the detailed mechanisms still remain unexplained. The detailed role of autonomic dysfunction in patients with IBS requires further evaluation.

\section{References}

Abe, M., Ando, Y., Higashi, K. and Kano, T. (1996). Non-neurogenic periodic fluctuations in heart rate and vasomotion appearing in familial amyloid polyneuropath (FAP) type 1 (Met30). J. Auton. Nerv. Syst. 60: 71-75.

Adeyemi, E.O.A., Desai, K.D., Towsey, M. and Ghista, D. (1999). Characterization of autonomic dysfunction in patients with irritable bowel syndrome by means of heart rate variability studies. Am. J. Gastroenterol. 94: 816-823.

Aggarawal, A., Cutts, T.F., Abell, T.L., Cardoso, S., Familoni, B., Bremer, J. and Karas, J. (1994). Predominant symptoms in irritable bowel syndrome correlate with specific autonomic nervous abnormalities. Gastroenterol. 106: 945-950.

Bockus, H.L., Bank, J. and Wilkinson, S.A. (1928). Neurogenic mucous colitis. Am. J. Med. Sci. 176: 813-829.

Bharucha, A.E., Camilleri, M., Low, P.A. and Zinsmeister, A.R. (1993). Autonomic dysfunction in gastrointestinal motility disorders. Gut 34: 397-401.

Buysschaert, M., Donckier, J., Dive, A., Ketelslegers, J.M. and Kambert, A.E. (1985). Gastric acid and pancreatic polypeptide responses to sham feeding are impaired in diabetic subjects with autonomic neuropathy. Diabetes 34: 1181-1185.

Chang, L., Mayer, E.A., Labus, J.S., Schmulson, M., Lee, O.Y., Olivas, T.I., Stains, J. and Naliboff, B.D. (2006). Effect of sex on perception of rectosigmoid stimuli in irritable bowel syndrome. Am. J. Physiol. 291: R277-R284.

Chang, L. and Heitkemper, M.M. (2002). Gender differences in irritable bowel syndrome. Gastroenterol. 123: $1686-1701$.

Drossman, D.A., Powel, D.W. and Sessions, J.T. (1977). The irritable bowel syndrome. Gastroenterol. 73: 811-822.

Drossman, D.A., Camilleri, M., Mayer, E.A. and Whitehead, W.E. (2002). AGA technical review on irritable bowel syndrome. Gastroenterol. 123: 2108-2131.

Elenkov, U., Wilder, R.L. and Chrousos, G.P. (2000). The sympathetic nerve-an integrative interface between two supersystems: the brain and immune system. Pharmacol. Rev. 52: 585-638.

Elsenbruch, S. and Orr, W.C. (2001). Diarrhea- and constipation-predominant IBS patients differ in postprandial autonomic and cortisol responses. Am. J. Gastroenterol. 96: 460-466.

Emmanuel, A.V. and Kamm, M.A. (2000). Laser Doppler flowmetry as a measure of extrinsic colonic 
innervation in functional bowel disease. Gut 46: 212-217.

Esler, M.D. and Goulston, K.J. (1973). Levels of anxiety in colonic disorders. N. Engl. J. Med. 288: 12-16. Everhart, J.E. and Renault, P.F. (1991). Irritable bowel syndrome in office based practice in the United States. Gastroenterol. 100: 998-1005.

Hansen, M.B. (2003). The enteric nervous system I: organization and classification. Pharmacol. Toxicol. 92: 105-113.

Heitkemper, M., Jarrett, M., Cain, K., Shaver, J., Bond, E., Woods, N.F. and Walker, E. (1996). Increased urine catecholamines and cortisol in women with irritable bowel syndrome. Am. J. Gastroenterol. 5: 906-913.

Iovino, P., Azpiroz, F., Domingo, E. and Malagelada, J.R. (1995). The symptomatic nervous system modulates perception and reflex responses to gut distension in human. Gastroenterol. 108: 680-686.

Karling, P., Nyhlin, H., Wiklund, U., Sjöberg, M., Olofsson, B.O. and Bjerle, P. (1998). Spectral analysis of heart rate variability in patients with irritable bowel syndrome. Scand. J. Gastroenterol. 33: 572576.

Longstreth, G.F. and Wolde-Tsadik, G. (1993). Irritable bowel-type symptoms in HMO examinees. Prevalence, demographics, and clinical correlates. Dig. Dis. Sci. 38: 1581-1589.

Malagelada, J.-R. and Azpiroz, F. (1989). Determinants of gastric emptying and transit in the small intestine. In: Handbook of Physiology, Section 6: Tthe Gastrointestinal System. Volume 1: Motility and Circulation, 2nd ed., eds. by S.G. Schultz, J.D. Wood, B.B. Rauner, American Physiological Society, Bethesda, MD, pp. 909-937.

Mayer, E.A. and Gebhart, G.F. (1994). Basic and clinical aspects of visceral hyperalgia. Gastroenterol. 107: 271-293.

Mayer, E.A., Berman, S., Chang, L. and Naliboff, B.D. (2004). Sex-based differences in gastrointestinal pain. Eur. J. Pain 8: 451-463.

Naliboff, B.D., Berman, S., Chang, L., Derbyshire, S.W., Suyenobu, B., Vogt, B.A., Mandelkern, M. and Mayer, E.A. (2003). Sex-related difference in IBS patients: central processing of visceral stimuli. Gastroenterol. 124: 1738-1747.

Pan, G., Lu, S., Ke, M., Han, S., Guo, H. and Fang, X. (2000). Epidemiologic study of the irritable bowel syndrome in Beijing: stratified randomized study by cluster sampling. Chin. Med. J. 113: 35-39.

Raamat, R., Jagomagi, K. and Kingisepp, P.-H. (2002). Simultaneous recording of fingertip skin blood flow changes by multiprobe laser Doppler flowmetry and frequency-corrected thermal clearance. Microvasc. Res. 64: 214-219.

Robert, J.J.T., Elsenbruch, S. and Whitehead, W.C. (2006). Sleep-related autonomic disturbances in symptom subgroups of women with irritable bowel syndrome. Dig. Dis. Sci. 51: 2121-2127.

Shiihara, Y., Hirota, A. and Kibayashi, Y. (1999). Skin blood flow responses during sleep by laser Doppler flowmetry. Psychiat. Clin. Neurosci. 53: 117-119.

Spanziani, R., Bayati, A., Redmond, K., Bajaj, H., Bienenstock, J., Collins, S.M. and Kamath, M.V. (2008). Vagal dysfunction in irritable bowel syndrome assessed by rectal distension and baroreceptor sensitivity. Neurogastroenterol. Motil. 20: 336-342.

Tanaka, T., Manabe, N., Hata, J., Kusunoki, H., Ishii, M., Sato, M., Kamada, T., Shiotani, A. and Haruma, K. (2007). Characterization of autonomic dysfunction in patients with irritable bowel syndrome using fingertip blood flow. Neurogastroenterol. Motil. 20: 498-504.

Tillish, K., Mayer, E.A., Labus, J.S., Stains, J., Chang, L. and Naliboff, B.D. (2005). Sex specific alterations in autonomic function among patients with irritable bowel syndrome. Gut 54: 1396-1401.

van Orshoven, N.P., Andriesse, G.I., van Schelven, L.J., Smout, A.J., Akkermans, L.M. and Oey, P.L. (2006). Subtle involvement of the parasympathetic nervous system in patients with irritable bowel syndrome. Clin. Auton. Res. 16: 33-39.

Viramontes, B., Camilleri, M., McKinzie, S., Pardi, D.S., Burton, D. and Thomforde, G.M. (2001). Gender-related differences in slowing colonic transit by a $5-\mathrm{HT}_{3}$ antagonist in subjects with diarrhea-predominant irritable syndrome. Am. J. Gastroenterol. 96: 2671-2679. 